\title{
Deep strong light-matter coupling in plasmonic nanoparticle crystals
}

Niclas S. Mueller, ${ }^{1}$ Yu Okamura, ${ }^{1}$ Bruno G. M. Vieira, ${ }^{1,2}$ Sabrina Juergensen, ${ }^{1}$ Holger Lange, ${ }^{3}$ Eduardo B. Barros, ${ }^{2}$ Florian Schulz, ${ }^{3}$ and Stephanie Reich*1

${ }^{1}$ Freie Universität Berlin, Department of Physics, Arnimallee 14, 14195 Berlin, Germany.

${ }^{2}$ Universidade Federal do Ceará, Department of Physics, Fortaleza, Ceará, 60455-760 Brazil.

${ }^{3}$ Institute of Physical Chemistry, University of Hamburg, Martin-Luther-King-Platz 6, 20146 Hamburg, Germany.

(Dated: March 31, 2020)

\begin{abstract}
In the regime of deep strong light-matter coupling, the coupling strength exceeds the materials transition energies ${ }^{1-3}$. This fundamentally changes materials properties ${ }^{4,5}$ : For example, the ground state of the system contains virtual photons and the internal electromagnetic field gets redistributed by the photon self interaction ${ }^{1,6}$. So far, no electronic excitation of a material has shown such strong coupling to free-space photons. Here we show that three-dimensional crystals of plasmonic nanoparticles are materials with deep strong coupling under ambient conditions, if the particles are ten times larger than the interparticle gaps. The experimental Rabi frequencies $(1.9-3.3 \mathrm{eV})$ of face-centered cubic crystals of $25-60 \mathrm{~nm}$ gold nanoparticles exceed their plasmon energy by up to $180 \%$. We show that the continuum of photons and plasmons hybridize into polaritons that violate the rotating-wave approximation. The coupling leads to a breakdown of the Purcell effect and increases the radiative polariton lifetime. We envision metallic and semiconducting nanoparticles as building blocks for an entire class of materials with extreme light-matter interaction. They will have applications in nonlinear optics, the search for cooperative effects and novel ground states, polariton chemistry, and quantum technology ${ }^{4,5}$.
\end{abstract}


Light is usually understood as an external perturbation to materials; electromagnetic modes are neglected in materials modeling. This approach works well in most cases, because the interaction of light and materials is weak. With increasing light-matter interaction, however, we reach a regime where the electromagnetic states fundamentally change the physical and chemical properties of a material ${ }^{4,5,7}$. The interaction may be quantified by the Rabi frequency $\Omega_{R}$ that measures how a two-level system periodically exchanges energy with a photon field ${ }^{4,5}$. Quantum optics defines distinct coupling regimes depending on how $\Omega_{R}$ compares to the transition frequency and lifetime. The classification highlights the relative contributions to light-matter coupling ${ }^{4,5}$ : When $\Omega_{R}$ becomes comparable to the transition frequency $\omega_{e}$ (ultrastrong coupling, $\left.\Omega_{R} \geq 0.1 \omega_{e}\right)$, the counter-rotating terms of the light-matter Hamiltonian become sizable, creating virtual excitations in the system ground state ${ }^{1,6,8}$. In the ultimate limit of light-matter coupling the interaction frequency $\Omega_{R}$ exceeds the energy of the excited state $\omega_{e}{ }^{4,5}$. In this deep strong coupling regime light and matter are expected to decouple due to the self-interaction energy of the photons, which leads to a breakdown of the Purcell effect ${ }^{6}$. Surprisingly, the regime of deep strong coupling is perturbative, although with switched roles compared to weak coupling: Lightmatter interaction dominates the energy scale and the material excitations are reduced to perturbations ${ }^{9}$. Light-matter coupling proceeds through the vacuum fluctuations of the electromagnetic field, which means that in the deep strong coupling regime high-field processes of non-linear optics will occur at low photon flux with unit efficiency ${ }^{10}$. Novel non-linear processes are expected, for example, the collective emission of a single photon by multiple atoms ${ }^{11}$. Deep strong coupling modifies the rate of chemical reactions and reaction coordinates ${ }^{7,12}$. Another exciting application is quantum information processing that may use new protocols, e.g., for ultra-fast quantum computation and protected qubits ${ }^{5,13,14}$.

Ultrastrong and deep strong light-matter coupling have been realized on various molecular and solid-state platforms ${ }^{4,5}$. The coupling regime where the counter-rotating and diamagnetic terms become dominant $\left(\Omega_{R} \geq 0.5 \omega_{e}\right)$, however, has been restricted to Landau states and superconducting circuits ${ }^{4,5}$. With few exceptions ${ }^{15}$, their Rabi splitting was on the order of $\mu \mathrm{eV}$ to $\mathrm{meV}$ and only emerged at cryogenic temperatures. Such devices couple discrete photonic and materials states. Although the deep strong coupling of an artificial atom to an electromagnetic continuum was realized recently ${ }^{16}$, a system that couples a continuum of materials excitations to free-space photons in the strongest regimes remains missing. Continuous systems will expand the realm and applicability of deep strong light-matter coupling, for example, for broadband cloaking and to replace dielectric metamaterials ${ }^{17,18}$. 
Here we propose and realize three-dimensional crystals of plasmonic nanoparticles as materials for deep strong light-matter coupling under ambient conditions. The collective transverse plasmon mode of the crystal hybridizes with the continuum of photonic states into three-dimensional polaritons with $\Omega_{R}$ approaching twice the plasmon frequency.

\section{PLASMON POLARITONS IN NANOPARTICLE CRYSTALS}

Metal nanoparticles absorb and scatter light through their localized surface plasmons, the collective oscillation of free electrons ${ }^{19,20}$. Their high oscillator strength makes them excellent building blocks for materials that strongly interact with light ${ }^{21-23}$. In an artificial crystal of metal nanoparticles, Fig. 1a, the dipole plasmons $\omega_{N P}$ couple through Coulomb interaction, creating a continuum of collective plasmonic states ${ }^{22,24}$, Methods and Extended Data Fig. 1. We introduce retardation through a propagating light field $\omega_{p t}(\mathbf{k})$, where $\mathbf{k}$ is the wave vector. $\omega_{p t}(\mathbf{k})$ couples to the transverse plasmon $\omega_{p l}^{t}(\mathbf{k})^{12,21,24}$, which is a unique state in nanoparticle crystals without a counterpart in bulk metals. Within a quantum mechanical formalism, the interaction may be described by a Hopfield Hamiltonian that yields hybridized light-matter polariton states $\omega_{p p}(\mathbf{k})^{25}$, Fig. 1b,

$$
\omega_{p p}^{4}(\mathbf{k})-\omega_{p p}^{2}(\mathbf{k})\left[\omega_{p t}^{2}(\mathbf{k})+\omega_{p l}^{2}(\mathbf{k})+4 \Omega_{R}^{2}\right]+\omega_{p t}^{2}(\mathbf{k}) \omega_{p l}^{2}(\mathbf{k})=0,
$$

where we abbreviated $\omega_{p l}^{t}=\omega_{p l} . \omega_{p t}=c|\mathbf{k}| / n_{\text {eff, }} c$ is the speed of light in vacuum and $n_{\text {eff }}$ the mean index of refraction if we replace the metal by vacuum, see Supplementary Table S1. An alternative approach models the plasmonic crystal through a classical oscillator with a giant oscillator strength, see Methods.
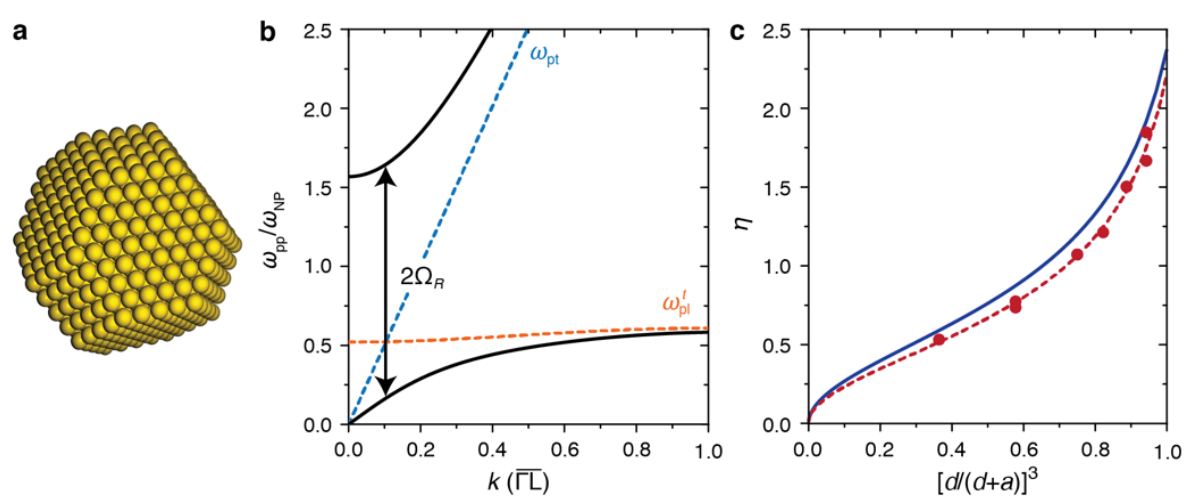

FIG. 1. Plasmon polaritons in metal nanoparticle fec crystals. (a) fec crystal of plasmonic nanoparticles with diameter $d=10-100 \mathrm{~nm}$. (b) Plasmon-polariton dispersion (black lines) along $\Gamma L$ for a Drude metal and $d=30 \mathrm{~nm}$ and $a=2 \mathrm{~nm}$. The orange line shows the transverse plasmon $\omega_{p l}^{t}(k)$ and the blue line the photon dispersion $\omega_{p t}(k)$. Light-matter coupling results in a level anticrossing with a large Rabi frequency $\Omega_{R}=1.4 \omega_{p l}^{t}$. (c) Normalized coupling strength $\eta=\Omega_{R} / \omega_{p l}^{t}$ as a function of $d$ and $a$ within the point-dipole approximation for a Drude metal (blue) and from FDTD simulations of gold (dots, the dashed line is a guide to the eye), $\varepsilon_{m}=1.96$, see Methods and Supplementary Table S1. 
The polariton dispersion consists of an upper and a lower branch arising from an anti-crossing of the photon and plasmon dispersions, Fig $1 \mathrm{~b}$. We calculate the Rabi splitting $2 \Omega_{R}$ between the two branches with a microscopic model, see Methods. For a face centered cubic (fcc) nanoparticle crystal with particle diameters $d=30 \mathrm{~nm}$ and interparticle gaps $a=2 \mathrm{~nm}$, lightmatter interaction results in a giant Rabi splitting, Fig $1 b$. The normalized coupling constant $\eta=$ $\Omega_{R} / \omega_{p l}^{t}=1.4$ is clearly in the deep strong regime. $\eta$ depends on the packing density and crystal structure. For an fcc crystal of spherical nanoparticles we obtain an expression for $\eta$ that depends on $d$ and $a$, Fig. 1c and Methods. The function agrees very well with numerical simulations of $\eta$ using the experimental dielectric function of gold, dots in Fig. 1c. Fcc crystals of gold nanoparticles enter the deep strong coupling regime for $a / d<0.1$. Prior fabrication protocols for plasmonic nanoparticle crystals were restricted to small crystallites ${ }^{26,27}$ or low packing densities $(a / d \gg 0.1)^{23,28-30}$, which has prevented the observation of deep strong coupling in such crystals.

\section{GOLD NANOPARTICLE CRYSTALS}

We synthesized densely packed fcc crystals of large gold nanoparticles $(d=25-59 \mathrm{~nm})$ with small gaps $(a \approx 1-4 \mathrm{~nm})$ using polystyrene as the nanoparticle ligand, see Methods. Polystyrenestabilized nanoparticles assemble into hexagonally packed monolayers at a liquid-liquid interface, Fig. $2 \mathrm{a}^{31-33}$. By reducing the reaction rate during self-assembly and increasing the nanoparticle concentration we synthesized nanoparticle fcc crystals, Fig. $2 b$ and c. Scanning electron microscopy (SEM) images show micron-sized crystals with little imperfection and excellent three-dimensional order, Fig. 2 and Extended Data Fig. 2. We found the number of crystal layers $N$ via optical microscopy in transmission for $N \leq 5$, Fig. 2 d, and by counting the scattering edges in dark-field images for thicker crystals, Fig. $2 \mathrm{e}^{33,34}$.

The optical response of the nanoparticle crystals demonstrates the presence of plasmon polaritons with deep strong light-matter coupling, Fig. 3. A series of polariton peaks stems from the absorption of photons in the infrared and lower visible energy range $(<1.7 \mathrm{eV})$, Fig. 3a, before light gets reflected above the polariton band edge $(>1.7 \mathrm{eV})$, Fig. 3c. At $2.3 \mathrm{eV}$ the interband transitions of gold set in and absorb any incoming light ${ }^{19,20}$. To quantify light-matter coupling, we extract the polariton dispersion from the optical spectra. The extrema in the spectra arise from open cavity boundary conditions, Fig. 3b. Each determines a pair of polariton frequency $\omega_{p p}$ and wavelength $\lambda_{p p}$, see Methods. The measured lower polariton branches of five nanoparticle crystals with $d=25-59 \mathrm{~nm}$, dots in Fig. 3d, show the characteristic bending for large $\eta$. We fit the dispersions using the Hopfield Hamiltonian treating $\Omega_{R}$ and $\omega_{p l}^{t}$ as free parameters, lines in Fig. 3d. Four of the five nanoparticle crystals are well in the deep strong 
coupling regime $\eta>1$, see Table I. The upper polariton branches are inaccessible optically, because they start above the onset of the gold interband transitions, see $\omega_{p l}^{l}$ in Table I. This branch should be observable in aluminium nanoparticle crystals using ultraviolet excitation, Extended Data Fig. 3.
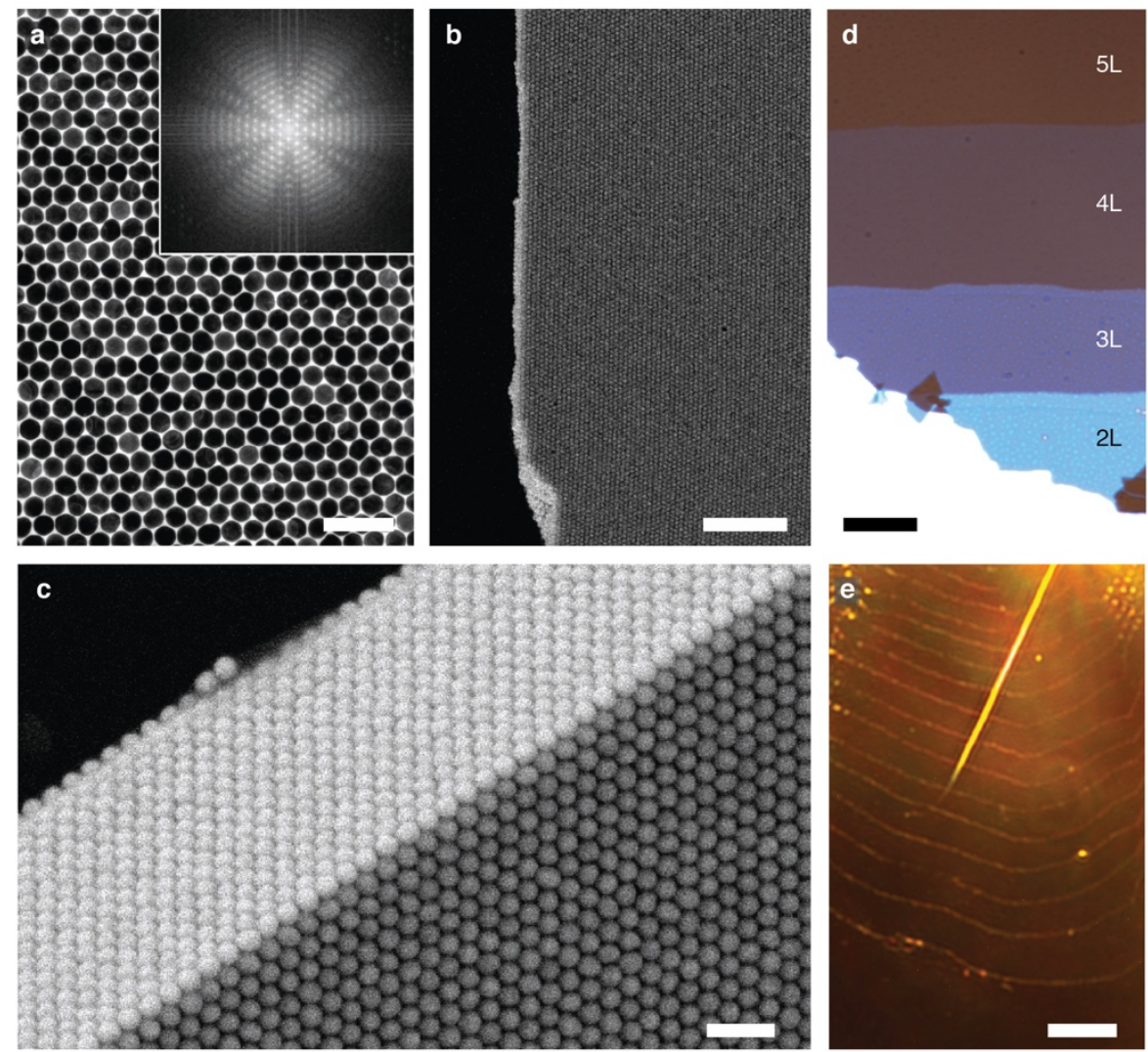

FIG. 2. Synthesis of gold nanoparticle crystals. (a) TEM image of a nanoparticle monolayer and Fourier transformed image as inset. (b) SEM image of a crystal with size $>6 \mu \mathrm{m}$. (c) Edge view of a nanoparticle crystal showing two (111) faces of fcc. (d) Transmission optical microscopy image of $N=2-5$ nanoparticle layers, see labels. (e) Dark field microscopy image up to $N=23$. The bright lines are layer edges that scatter light. The scale bars are (a) $200 \mathrm{~nm}$, (b) $1 \mu \mathrm{m}$, (c) $200 \mathrm{~nm}$, and (d,e) $10 \mu \mathrm{m}$. Panels (a)(c) show crystals with $d=59 \mathrm{~nm}$, (d) and (e) $d=30 \mathrm{~nm}$.

Deep strong coupling in plasmonic nanoparticle crystals stands out by its exceptional normalized coupling strength and the large absolute values of the Rabi frequency, Fig. 3e. The highest normalized coupling (1.83) for the $59 \mathrm{~nm}$ nanoparticles greatly exceeds the values reported in optimized systems of Landau polaritons $(1.43)^{2}$ and superconducting circuits $(1.34)^{3}$. The large absolute Rabi frequency ensures that our system is simultaneously in the deep strong $\left(\Omega_{R}>\omega_{p l}^{t}\right)$ and the strong coupling regime, i.e., $\Omega_{R}$ exceeds any losses in the material ${ }^{35}$. We estimate the cooperativity factor as $C \approx 4 \Omega_{R}^{2} / \gamma_{p p}^{2}=5,500$ from the width of the polariton peaks $\hbar \gamma_{p p}=90 \mathrm{meV}(d=59 \mathrm{~nm} \text {, see below })^{5,8}$. The Rabi frequency may be tailored by varying the crystal parameters, which let us realize $\eta=0.95-1.83$. A further increase in packing density will 
push the coupling towards the upper bound $\eta=2.2$ predicted in Fig. 1c. Exceeding this limit will be possible by changing the dielectric background, optimizing the oscillator strength per nanoparticle, and perhaps by using other crystal structures.
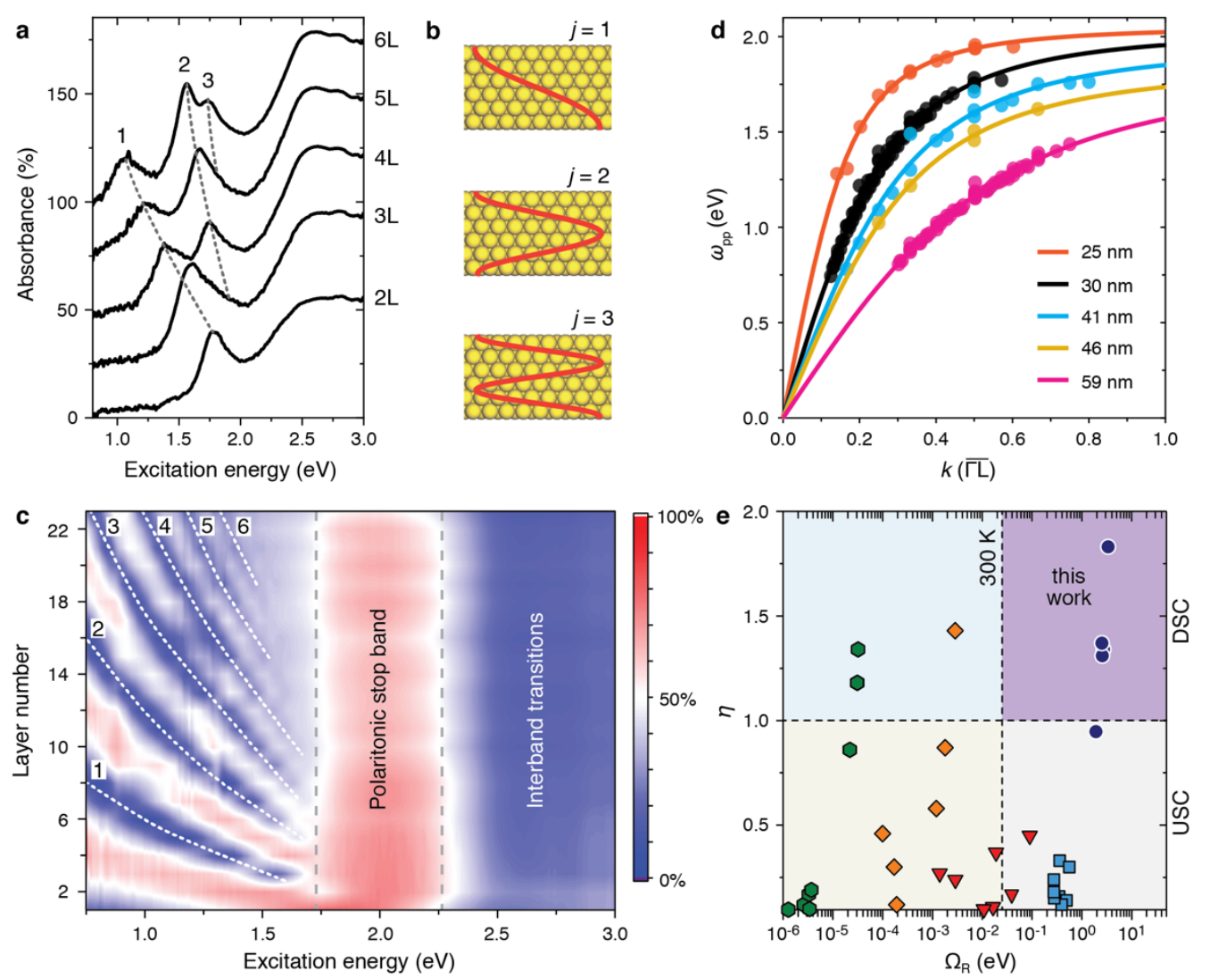

FIG. 3. Deep strong light-matter coupling in nanoparticle crystals. (a) Absorption spectra of the 30 $\mathrm{nm}$ gold nanoparticle crystal for two to six layers, offset by $25 \%$ increments. The peaks correspond to standing waves inside the crystal as sketched in (b). (c) Reflectance as a function of excitation energy and layer number. Dashed lines and labels indicate $j=2 h / \lambda_{p p}$. The onsets of the polaritonic stop band and the interband transitions are highlighted by grey dashed lines. (d) Measured lower polariton branch for crystals with $d=25 \mathrm{~nm}$ - orange dots, $30 \mathrm{~nm}$ - black, $41 \mathrm{~nm}$ - blue, $46 \mathrm{~nm}$ - yellow, and $59 \mathrm{~nm}$ - pink. The solid lines are fits with Eq. (1) assuming that the coupling strength is constant with layer number, see discussion in Methods; Table I lists the fitting parameters. (e) Comparison of $\eta$ and $\Omega_{R}$ measured in this work (dots) and superconducting circuits (hexagons), Landau polaritons (diamonds), intersubband polaritons (triangles), and organic molecules (squares) taken from the studies in Table 1 of Ref. 4. The horizontal dashed line marks the onset of deep strong light-matter coupling; the vertical dashed line shows the thermal energy at room temperature.

Light-matter coupling strongly reduces the phase $v_{p h}$ and group velocity of light $v_{g r}$ inside the nanoparticle crystal, Table I. The reduction in wavelength by an order of magnitude implies an enhancement of $10^{3}$ for dipole and $10^{5}$ for quadrupole transitions that get excited by the polariton near-field compared to far-field excitation ${ }^{36}$. Crystals with embedded molecules may show forbidden transitions through the shrinking of light ${ }^{36}$. Group and phase velocity in the crystals 
depend sensitively on $d, a$, and $\varepsilon_{m}$, making them a potential material for manipulating light at the nanoscale. They may borrow concepts from photonic crystals and realize them with a smaller footprint due to the drastically reduced wavelength ${ }^{18}$.

TABLE I. Measured light-matter coupling

\begin{tabular}{ccccccccc}
\hline \hline$d(\mathrm{~nm})$ & $a(\mathrm{~nm})$ & $\eta$ & $\Omega_{R}(\mathrm{eV})$ & $\omega_{p l}^{t}(\mathrm{eV})$ & $\omega_{p l}^{l}(\mathrm{eV})$ & $\lambda_{p p}(0.8 \overline{\Gamma L})(\mathrm{nm})$ & $v_{p h} / c$ & $v_{g r} / c$ \\
\hline 25 & 3 & 0.95 & 1.94 & 2.05 & 4.39 & 58 & 0.09 & $3 \cdot 10^{-3}$ \\
30 & 4 & 1.34 & 2.72 & 2.04 & 5.80 & 69 & 0.11 & $9 \cdot 10^{-3}$ \\
41 & 3 & 1.31 & 2.57 & 1.96 & 5.49 & 90 & 0.13 & $1.7 \cdot 10^{-2}$ \\
46 & 2 & 1.4 & 2.5 & 1.8 & 5.4 & 100 & 0.1 & $2 \cdot 10^{-2}$ \\
59 & 1 & 1.83 & 3.35 & 1.83 & 6.94 & 123 & 0.14 & $4.7 \cdot 10^{-2}$ \\
\hline
\end{tabular}

Rabi frequency $\Omega_{R}$, transverse plasmon frequency $\omega_{p l}^{t}$, and normalized coupling strength $\eta$ measured on five nanoparticle crystals. $d$ and $a$ were estimated from TEM images. $\omega_{p l}^{l}$ is the longitudinal plasmon frequency calculated from the fits. The table lists the wavelength $\lambda_{p p}$ at $k=$ $0.8 \overline{\Gamma L}$ together with the phase velocity $v_{p h} / c=\lambda_{p p} / \lambda_{0}\left(\lambda_{0}\right.$ is the light wavelength in vacuum) and group velocity $v_{g r} / c=1 / c \cdot \partial \omega_{p p} / \partial k$ at this wavelength. The uncertainty is in the last digit.

\section{PURCELL EFFECT IN DEEP STRONG COUPLING}

Among the novel effects that have been predicted for deep strong light-matter coupling is the breakdown of the Purcell effect ${ }^{6}$. The Purcell effect states that radiative damping increases through light-matter coupling, $\gamma_{\text {rad }} \propto \Omega_{R}^{2}$ for $\Omega_{R} \ll \omega_{e}$ (here, $\left.\omega_{p l}^{t}\right)^{37,38}$. It plays a key role in engineering optical emission, e.g., for near-field enhanced spectroscopy, non-linear optics, and lasing7. The Hopfield Hamiltonian gives insight into which terms of light-matter coupling decrease the radiative damping for $\eta>1^{6}$. To demonstrate this, we first analyse the contribution of the counter-rotating terms and the photon self-interaction energy to the polariton dispersion ${ }^{4,5}$. A two-mode version of the Hopfield Hamiltonian reads ${ }^{25}$, see Supplementary Information,

$$
\mathcal{H}=\hbar \omega_{p t}(k) \hat{a}^{\dagger}(k) \hat{a}(k)+\hbar \omega_{p l}^{t}(k) \hat{b}^{\dagger}(k) \hat{b}(k)+\mathcal{H}_{\text {int }}+\mathcal{H}_{A^{2}}
$$

with the interaction Hamiltonian

$$
\mathcal{H}_{\text {int }}=i \hbar \Omega_{R} \sqrt{\frac{\omega_{p l}^{t}(k)}{\omega_{p t}(k)}}\left[\hat{a}^{\dagger}(-k)+\hat{a}(k)\right]\left[\hat{b}(-k)-\hat{b}^{\dagger}(k)\right],
$$

and the photon self-interaction $\left(\mathrm{A}^{2}\right.$ term)

$$
\mathcal{H}_{A^{2}}=\frac{\hbar \Omega_{R}^{2}}{\omega_{p t}(k)}\left[\hat{a}^{\dagger}(-k)+\hat{a}(k)\right]\left[\hat{a}^{\dagger}(k)+\hat{a}(-k)\right] .
$$


$\hat{a}^{\dagger}(k)$ and $\hat{a}(k)$ are creation and annihilation operators of a photon $\omega_{p t}(k)=c k / n_{\text {eff }}$ and $\hat{b}^{\dagger}(k)$ and $\hat{b}^{\dagger}(k)$ of the collective transverse plasmon $\omega_{p l}^{t}(k)$ (taken as constant). A fit with the full Hamiltonian excellently describes the measurements, Figs. 3d and 4a. When forcing all counterrotating terms $-\hat{a}^{\dagger} \hat{b}^{\dagger}, \hat{a} \hat{b}, \hat{a}^{\dagger} \hat{a}^{\dagger}$, and $\hat{a} \hat{a}$ - to zero, the polariton energy becomes finite for $k \rightarrow$ 0 and increases by the vacuum Bloch-Siegert shift $\left|\Delta_{V B S}\right|=100-900 \mathrm{meV}$, orange line in Fig. 4a and Extended Data Fig. $4 \mathrm{a}^{8}$. Even when treating $\Omega_{R}$ and $\omega_{p l}^{t}$ as free parameters, it was impossible to fit the data with a dispersion that neglects the counter-rotating contributions, see Extended Data Fig. 4b: The best fit produces optical resonances, orange arrows in Fig. 4b, that disagree strongly with the measured peak positions. We obtain similar disagreement, Fig. 4a and $\mathrm{b}$, without the $A^{2}$ term (Dicke model, pink line) and for the rotating-wave approximation (RWA, blue). The counter-rotating contributions to light-matter coupling populate the system ground state with virtual excitations ${ }^{1}$. The population exceeds unity for small wave vectors, Extended Data Fig. 5. The virtual photons may be converted into real states when turning off light-matter coupling non-adiabatically ${ }^{1,4,39}$.
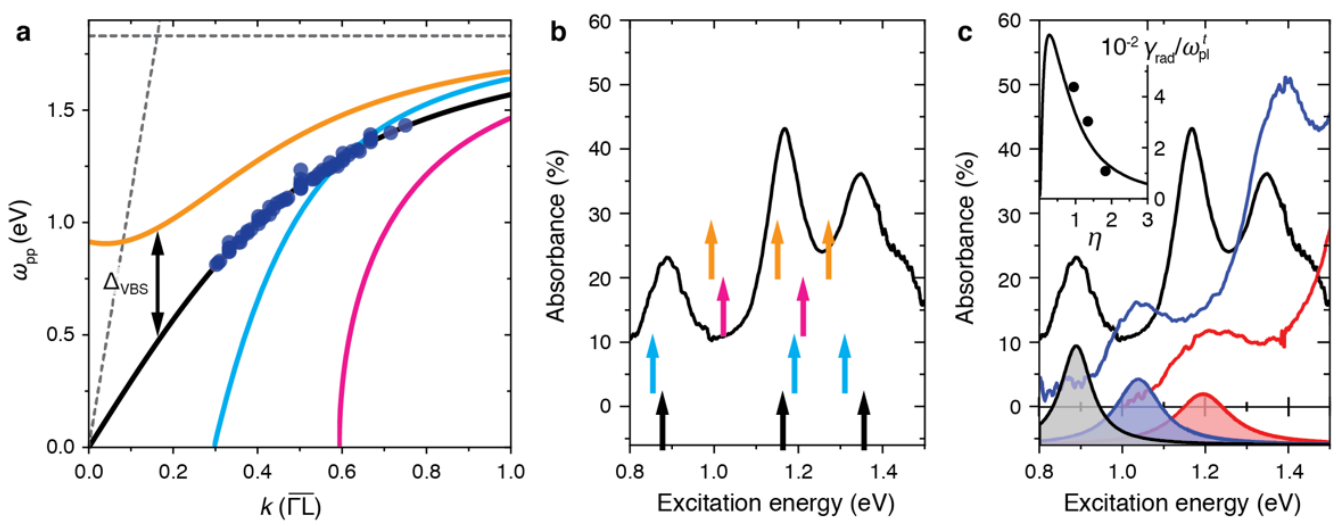

FIG. 4. Counter-rotating and photon self-interaction terms in deep strong coupling. (a) Black line polariton dispersion $(d=59 \mathrm{~nm})$ with the Hopfield model, orange - without the counter-rotating contributions, pink - Dicke model, and blue - RWA that neglects both the counter-rotating and the $A^{2}$ terms. The arrow indicates the vacuum Bloch-Siegert shift $\left(\Delta_{V B S}=510 \mathrm{meV}\right)$ under resonance conditions $\omega_{p t}=\omega_{p l}^{t}$. The dashed lines are the bare photon and plasmon dispersion. (b) Absorption spectrum for $d=$ $59 \mathrm{~nm}$ compared to the peak positions predicted within the Hopfield model (black arrows), without counter-rotating contributions (orange), the Dicke model (pink), and RWA (blue). The predictions were made from the best fits of the models to the experimental data, Extended Data Fig. 4b. (c) Increase in intensity and decrease in line width of the absorption peak due to the $A^{2}$ term. Absorption spectra and fit to the $j=2$ peak for $300 \mathrm{~nm}$ thick crystals of $d=59 \mathrm{~nm}$ (black line, FWHM of $90 \mathrm{meV}$ ), $d=30 \mathrm{~nm}$ (blue, $132 \mathrm{meV}$ ), and $d=25 \mathrm{~nm}$ (red, $160 \mathrm{meV}$ ) nanoparticles. Inset: Symbols show the radiative damping $\gamma_{\text {rad }}$ obtained after subtracting an intrinsic contribution $\hbar \gamma_{i}=70 \mathrm{meV}$ (gold, $1 \mathrm{eV}$, see Methods). The line is the behavior predicted for $\gamma_{i}=\gamma_{N P}=0.1 \omega_{p l}^{t}$ ( $\gamma_{N P}$ is the radiative line width of an isolated nanoparticle $)^{6}$. 
The Purcell effect depends on the density of electromagnetic states and their spatial overlap with the excited material states ${ }^{6,37}$. The strong dielectric contrast of metals and dielectrics confines the electric field to the particle gaps for $\omega_{p t}<\omega_{p l}^{t}$. To minimize the large photon selfinteraction energy, the $A^{2}$ term rearranges the electric field and partly pushes it into the metal ${ }^{6}$. This shifts the plasmon resonance to higher energies preventing the superradiant phase transition expected within the Dicke model ${ }^{4,5}$. Most strikingly, we observe a decrease in radiative damping, which follows the scaling predicted from the breakdown of the Purcell effect ${ }^{6}$, Fig. 4c. The full width at half maximum (FWHM) of the polariton peak decreased by a factor of two when increasing $\eta$ from 0.95 to 1.83 . The drop in emission is accompanied by a raise in integrated absorption intensity $(10 \%$, Fig. $4 \mathrm{c})$ due to the stronger overlap between the electric field and the metal electrons. The calculated optical transmission fully captures this counter-intuitive behavior, showing an increasing polariton absorption combined with a narrowing line width in the lower polariton branch, Extended Data Fig. 6. The Purcell effect in plasmonic nanoparticle crystals depends on polariton wave vector, plasmon energy, and crystal thickness in addition to the Rabi frequency, which opens ways to engineer the radiative decay for a target application.

\section{DISCUSSION}

Deep strong light-matter coupling emerged naturally for the transverse plasmon in fcc nanoparticle crystals with record-high normalized coupling strength. The large Rabi frequency made the phenomena robust against thermal excitation and polaritons observable at room temperature. Quantum optics sets an inspiring framework to understand the properties of plasmonic crystals; the theory predicts physical phenomena like the vanishing Purcell effect and quantum effects like squeezed light and virtual excitations, Extended Data Fig. 5 and Ref. 40. A classical way for modeling the optical properties of the crystals is as an artificial material with a giant oscillator strength $f>10$, see Methods. Nanoparticle crystals are a versatile platform for engineering light-matter interaction. They may be synthesized from nanoparticles of various shape, other metals, and doped semiconductors. Mixed crystals of metals and quantum dots will simultaneously couple excitons and plasmons to photons. Embedding molecules in a material with deep strong coupling will tailor their excited state dynamics and may realize vibrational strong coupling. The potential applications of our work range from fundamental studies of quantum electrodynamics over quantum technologies, nanophotonics, and non-linear optics to analytics in chemistry and life sciences. 


\section{REFERENCES}

[1] Ciuti, C., Bastard, G. \& Carusotto, I. Quantum vacuum properties of the intersubband cavity polariton field. Phys. Rev. B 72, 115303 (2005).

[2] Bayer, A. et al. Terahertz light-matter interaction beyond unity coupling strength. Nano Lett. 17, 6340-6344 (2017).

[3] Yoshihara, F. et al. Superconducting qubit-oscillator circuit beyond the ultrastrong-coupling regime. Nat. Phys. 13, 44 (2016).

[4] Frisk Kockum, A., Miranowicz, A., De Liberato, S., Savasta, S. \& Nori, F. Ultrastrong coupling between light and matter. Nat. Rev. Phys. 1, 19-40 (2019).

[5] Forn-Díaz, P., Lamata, L., Rico, E., Kono, J. \& Solano, E. Ultrastrong coupling regimes of light-matter interaction. Rev. Mod. Phys. 91, 025005 (2019).

[6] De Liberato, S. Light-matter decoupling in the deep strong coupling regime: The breakdown of the Purcell effect. Phys. Rev. Lett. 112, 016401 (2014).

[7] Baranov, D. G., Wersäll, M., Cuadra, J., Antosiewicz, T. J. \& Shegai, T. Novel nanostructures and materials for strong light-matter interactions. ACS Photonics 5, 24-42 (2018).

[8] Li, X. et al. Vacuum Bloch-Siegert shift in Landau polaritons with ultra-high cooperativity. Nat. Photon. 12, 324 (2018).

[9] Rossatto, D. Z., Villas-Bôas, C. J., Sanz, M. \& Solano, E. Spectral classification of coupling regimes in the quantum Rabi model. Phys. Rev. A 96, 013849 (2017).

[10] Kockum, A. F., Miranowicz, A., Macrì, V., Savasta, S. \& Nori, F. Deterministic quantum nonlinear optics with single atoms and virtual photons. Phys. Rev. A 95, 063849 (2017).

[11] Garziano, L. et al. One photon can simultaneously excite two or more atoms. Phys. Rev. Lett. 117, 043601 (2016).

[12] Hutchison, J. A., Schwartz, T., Genet, C., Devaux, E. \& Ebbesen, T. W. Modifying chemical landscapes by coupling to vacuum fields. Angew. Chem. Int. Ed. 51, 1592-1596 (2012).

[13] Nataf, P. \& Ciuti, C. Protected quantum computation with multiple resonators in ultrastrong coupling circuit QED. Phys. Rev. Lett. 107, 190402 (2011).

[14] Romero, G., Ballester, D., Wang, Y. M., Scarani, V. \& Solano, E. Ultrafast quantum gates in circuit QED. Phys. Rev. Lett. 108, 120501 (2012).

[15] Askenazi, B. et al. Midinfrared ultrastrong light-matter coupling for THz thermal emission. ACS Photonics 4, 2550-2555 (2017).

[16] Forn-Díaz, P. et al. Ultrastrong coupling of a single artificial atom to an electromagnetic continuum in the nonperturbative regime. Nat. Phys. 13, 39 (2016). 
[17] Chen, P.-Y., Soric, J. \& Alù, A. Invisibility and cloaking based on scattering cancellation. Adv. Mater. 24, OP281-OP304 (2012).

[18] Joannopoulos, J. D., Johnson, S. G., Winn, J. N. \& Meade, R. D. Photonic Crystals: Molding the Flow of Light (Second Edition) (Princeton University Press, 2008), 2 edn.

[19] Maier, S. Plasmonics: Fundamentals and Applications (Springer, New York, 2007).

[20] Halas, N. J., Lal, S., Chang, W.-S., Link, S. \& Nordlander, P. Plasmons in strongly coupled metallic nanostructures. Chem. Rev. 111, 3913-3961 (2011).

[21] Yannopapas, V., Modinos, A. \& Stefanou, N. Optical properties of metallodielectric photonic crystals. Phys. Rev. B 60, 5359-5365 (1999).

[22] Huang, C.-P., Yin, X.-G., Wang, Q.-J., Huang, H. \& Zhu, Y.-Y. Long-wavelength optical properties of a plasmonic crystal. Phys. Rev. Lett. 104, 016402 (2010).

[23] Park, D. J. et al. Plasmonic photonic crystals realized through DNA-programmable assembly. PNAS 112, 977-981 (2015).

[24] Lamowski, S. et al. Plasmon polaritons in cubic lattices of spherical metallic nanoparticles. Phys. Rev. B 97, 125409 (2018).

[25] Hopfield, J. J. Theory of the contribution of excitons to the complex dielectric constant of crystals. Phys. Rev. 112, 1555-1567 (1958).

[26] Hanske, C. et al. Large-scale plasmonic pyramidal supercrystals via templated self-assembly of monodisperse gold nanospheres. J. Phys. Chem. C 121, 10899-10906 (2017).

[27] Matricardi, C. et al. Gold nanoparticle plasmonic superlattices as surface-enhanced Raman spectroscopy substrates. ACS Nano 12, 8531-8539 (2018).

[28] Lau, C. Y. et al. Enhanced ordering in gold nanoparticles self-assembly through excess free ligands. Langmuir 27, 3355-3360 (2011).

[29] Ross, M. B., Ku, J. C., Vaccarezza, V. M., Schatz, G. C. \& Mirkin, C. A. Nanoscale form dictates mesoscale function in plasmonic DNA-nanoparticle superlattices. Nat. Nanotechnol. 10, 453 (2015).

[30] Sun, L., Lin, H., Kohlstedt, K. L., Schatz, G. C. \& Mirkin, C. A. Design principles for photonic crystals based on plasmonic nanoparticle superlattices. PNAS 115, 7242-7247 (2018).

[31] Schulz, F., Tober, S. \& Lange, H. Size-dependent phase transfer functionalization of gold nanoparticles to promote well-ordered self-assembly. Langmuir 33, 14437-14444 (2017).

[32] Ye, X. et al. Structural diversity in binary superlattices self-assembled from polymer-grafted nanocrystals. Nat. Commun. 6, 10052 (2015).

[33] Mueller, N. S. et al. Dark interlayer plasmons in colloidal gold nanoparticle bi- and fewlayers. ACS Photonics 5, 3962-3969 (2018). 
[34] Mueller, N. S. et al. Direct optical excitation of dark plasmons for hot electron generation. Faraday Discuss. 214, 159-173 (2019).

[35] De Liberato, S. Virtual photons in the ground state of a dissipative system. Nat. Commun. 8, 1465 (2017).

[36] Rivera, N., Kaminer, I., Zhen, B., Joannopoulos, J. D. \& Soljačić, M. Shrinking light to allow forbidden transitions on the atomic scale. Science 353, 263-269 (2016).

[37] Purcell, E. M., Torrey, H. C. \& Pound, R. V. Resonance absorption by nuclear magnetic moments in a solid. Phys. Rev. 69, 37-38 (1946).

[38] Ciuti, C. \& Carusotto, I. Input-output theory of cavities in the ultrastrong coupling regime: The case of time-independent cavity parameters. Phys. Rev. A 74, 033811 (2006).

[39] Günter, G. et al. Sub-cycle switch-on of ultrastrong light-matter interaction. Nature 458, 178-181 (2009).

[40] Artoni, M. \& Birman, J. L. Quantum-optical properties of polariton waves. Phys. Rev. B 44, 3736-3756 (1991). 


\section{ACKNOWLEDGEMENTS}

We are grateful to A. Knorr, G. Weick, and S. De Liberato for fruitful discussions and critical comments. We thank M. Wolf for the use of the supercontinuum light source. This work was supported by the European Research Council (ERC) under grant DarkSERS (772108). S.R. acknowledges the Focus Area NanoScale of Freie Universitaet Berlin. F.S. was supported by the DFG via the project SCHU 3019/2-1. H.L. and F.S. acknowledge support by the Cluster of Excellence 'Advanced Imaging of Matter' of the DFG (EXC 2056 - project ID 390715994). B.G.M.V. acknowledges the Coordenação de Aperfeiçoamento de Pessoal de Nivel Superior (CAPES) for financial support under the program PDSE (grant no. 88881.134611/2016-01) and Dahlem Research School (DRS). E.B.B. and B.G.M.V. acknowledge the financial support from CNPq, CAPES (finance code 001), and FUNCAP (PRONEX PR2-0101-00006.01.00/15).

\section{AUTHOR CONTRIBUTIONS}

S.R. and N.S.M. conceived and designed the research project. The gold nanoparticle crystals were synthesized by F.S. and H.L.; the characterization and optical experiments were performed by N.S.M., Y.O., F.S., and S.J.. The analytic theory and FDTD simulations were developed by N.S.M., B.G.M.V., E.B.B., and S.R.. The data were analyzed by N.S.M., F.S., and S.R.. S.R. and N.S.M. wrote the initial draft of the manuscript on which all co-authors commented.

\section{AUTHOR INFORMATION}

The authors declare no competing interests. Correspondence and requests for materials should be addressed to reich@physik.fu-berlin.de. 


\section{METHODS}

\section{Synthesis of gold nanoparticle crystals}

Gold nanoparticles with diameters $d=30,41$, and $46 \mathrm{~nm}$ were synthesized with the seeded growth protocol by Bastús et al. ${ }^{41}$ and functionalized with thiol-terminated polystyrene $\left(M_{n}=\right.$ 5,300 g/mol, Polymer Source, Montral, Canada) using a phase-transfer protocol that some of us presented recently ${ }^{31}$. Gold nanoparticles with $d=25$ and $59 \mathrm{~nm}$ were synthesized with the seeded growth protocol by Zheng et $a l^{42}$ scaled up by a factor of ten. $10 \mathrm{ml}$ of the CTAC (cetyltrimethylammonium chloride)-stabilized nanoparticles in water were purified and concentrated by repeated centrifugation $(d=25 \mathrm{~nm}: 5,000 \mathrm{~g} \times 12 \mathrm{~min}, 59 \mathrm{~nm}: 2,000 \mathrm{~g} \times 12 \mathrm{~min})$ to $100 \mu 1$ and injected into a solution of thiol-terminated polystyrene $\left(25 \mathrm{~nm}: M_{n}=5,300 \mathrm{~g} / \mathrm{mol}\right.$, $59 \mathrm{~nm}: M_{n}=11,500 \mathrm{~g} / \mathrm{mol}$, Polymer Source, Montreal, Canada) in tetrahydrofuran $(0.5 \mathrm{mM}, 2$ $\mathrm{ml})$. After reaction overnight, the solvent was removed under reduced pressure in a rotary evaporator, the residue solved in $2.0 \mathrm{ml}$ toluene, purified, and concentrated to $30 \mathrm{nM}(25 \mathrm{~nm})$ and $1.4 \mathrm{nM}(59 \mathrm{~nm})$ gold nanoparticle concentration by repeated centrifugation.

The synthesis of the gold nanoparticle crystals was performed with the method described by Murray and co-workers ${ }^{43}$ : Self-assembly during evaporation of toluene on a liquid subphase, namely $300 \mu 1$ diethylene glycol. The self-assembly was carried out in small wells (high density polyethylene, HDPE) that were covered with glass slides. We used $300 \mu 1$ of $30 \mathrm{~nm}(3.5 \mathrm{nM})$ and $41 \mathrm{~nm}(0.3 \mathrm{nM})$ gold nanoparticles in toluene, $250 \mu 1$ of $46 \mathrm{~nm}$ nanoparticles $(2.1 \mathrm{nM})$ and 100 $\mu 1$ of $25 \mathrm{~nm}(30 \mathrm{nM})$ and $59 \mathrm{~nm}$ nanoparticles (1.4 nM). After complete evaporation of the solvent (usually after 24-48 h) the formed crystals were carefully skimmed off with carbon coated TEM grids for TEM characterization or transferred on glass slides $(0.13-0.16 \mathrm{~mm}$ thickness, Carl Roth, Germany) for optical spectroscopy.

\section{Electron microscopy}

Transmission (TEM) and scanning electron microscopy (SEM) were carried out to characterize the samples on various length scales. TEM measurements were performed using a Jeol JEM-1011 instrument operating at $100 \mathrm{kV}$. The particle diameters and nearest neighbour distances were determined with the software Image J 1.50i. Extended Data Figure 7 shows an exemplary analysis for a monolayer of $d=59 \mathrm{~nm}$ nanoparticles. The nanoparticle diameters as determined by TEM were $59 \pm 2 \mathrm{~nm}$, which was obtained by analyzing $N_{N P}=216$ particles. For the other particles we obtained $25 \mathrm{~nm}-25 \pm 1 \mathrm{~nm}\left(N_{N P}=366\right), 30 \mathrm{~nm}$ nanoparticles $-30 \pm 3 \mathrm{~nm}$ $\left(N_{N P}=455\right), 41 \mathrm{~nm}$ nanoparticles $-41 \pm 3 \mathrm{~nm}\left(N_{N P}=651\right)$, and $46 \mathrm{~nm}$ nanoparticles $-46 \pm 4 \mathrm{~nm}$ $\left(N_{N P}=228\right)$. The interparticle distances were found from a nearest neighbour analysis, yielding 
$a=3 \mathrm{~nm}$ for the $25 \mathrm{~nm}$ nanoparticles, $4 \mathrm{~nm}$ for $30 \mathrm{~nm}, 3 \mathrm{~nm}$ for $41 \mathrm{~nm}, 2 \mathrm{~nm}$ for $46 \mathrm{~nm}$, and 1 $\mathrm{nm}$ for $59 \mathrm{~nm}$ nanoparticles. The accuracy of $a$ is limited by variations in diameter and deviations of the shape from the ideal sphere. The interparticle distance $a$ was evaluated for nanoparticle monolayers; distances may vary for thicker slabs, which we will study by scattering techniques in future.

SEM images of the nanoparticle crystal were taken with a Pioneer Two system from Raith. We used an acceleration voltage of $7 \mathrm{kV}$ and set the aperture size to $7.5 \mu \mathrm{m}$. The working distance was adjusted to $6.7 \mathrm{~mm}$. To detect the ejected electrons a standard secondary electron detector was used. The images were recorded on TEM grids, which were attached to a silicon substrate with silver glue.

\section{Optical microscopy and spectroscopy}

We used optical microscopy to assess the quality of the synthesized crystals and as a precharacterization tool for spectroscopy. Measuring the polariton spectra and dispersions in Fig. 3 required precise control over the number of stacked layers. We identified sample areas with stepwise increasing thickness that were ideal for our measurements. For thin crystals of less than six layers, we determined the thickness from the optical contrast in transmittance (Fig. 2d). For thicker crystals, we used the scattering of light from the layer edges to count the number of stacked layers. These were visible as lines in optical reflectance and dark-field images (Fig. 2e). We identified sample areas with step-wise increasing height up to $h=23$ layers $=0.60 \mu \mathrm{m}$ in a $d$ $=30 \mathrm{~nm}$ and $h=17$ layers $=0.84 \mu \mathrm{m}$ in a $d=59 \mathrm{~nm}$ crystal. The samples also contained much thicker crystals, but we needed an accurate measure of $h$ to determine the polariton dispersion.

The optical spectra of gold nanoparticle crystals were measured with a micro-absorbance spectrometer. For a detailed description of the setup we refer to Ref. 33. A supercontinuum laser (Fianium, SC-400-4) acts as the light source over a broad spectral range (450 - $2400 \mathrm{~nm}$ ). An optical microscope (Olympus, IX71) with home-made modifications focused the light onto the sample with a Leica HCX PL Fluotar $100 \times$ objective (NA =0.9). Note that due to the large index of refraction of the nanoparticle crystals $n_{p p}=4-10$ (see Table I), the effective NA inside the material $\mathrm{NA}_{e f f}=\mathrm{NA} / n_{p p}$ ranges from $\mathrm{NA}_{\text {eff }}=0.09-0.23$. The sample position was changed with a motorized $x y$ stage combined with a piezo stage (PI, P-542.2CD). The position of the laser spot on the sample was identified with a microscope camera in transmission or reflection. The laser power at the sample was kept below $100 \mu \mathrm{W}$.

The transmitted light was collected with an Olympus MPlan FL N BD $100 \times$ objective $(\mathrm{NA}=$ 0.9). An optical fibre (Ocean Optics, QP600-2-UV-BX for 450-900 nm and BIF600- VIS-NIR 
for 900-1600 nm) was used to guide the light to a spectrometer (Avantes, AvaSpec 3648 for 450$900 \mathrm{~nm}$ and AvaSpec NIR512 for 900-1600 nm), where it was dispersed and detected. As a reference for the transmission spectra we used the transmittance through the substrate at a place without nanoparticle crystals. The reflected light was separated from the incoming light by a beam splitter (ThorLabs, BSW26R for 450-900 nm and BSW29R for 900-1600 nm) and detected with the same fibres and spectrometers as for the transmitted light. The reflections from a silver mirror (Thorlabs, PF10-03-P01) served as a reference for the reflection measurements. The transmittance $T$ and reflectance $R$ spectra of the two spectral ranges were pinned together and the absorbance was calculated as $A=100 \%-T-R$.

The absorption spectra were fit with a cubic background and a series of Lorentzians to obtain the polariton frequencies. The crystal forms an open cavity for polaritons creating standing waves with wavelength $\lambda_{p p}=2 h / j$, where $j$ is integer, Fig. $3 b^{21-23,33}$. The wave vectors in units of the $\Gamma L$ distance are given by $k_{p p}=2 \pi / \lambda_{p p}=(j / N) \overline{\Gamma L}$, where $N$ is the layer number. In Fig. 3c we highlighted polaritons with $j=1-6$ that were used to relate the measured polariton energy with its wavelength $\lambda_{p p}$. That way we obtained the experimental polariton dispersions, shown as data points in Fig. 3 d.

\section{Finite-difference time-domain simulations}

We used the commercial finite-difference time-domain (FDTD) simulation package Lumerical FDTD solutions. We constructed the unit cell of a hexagonally close packed monolayer of metallic nanoparticles with diameters $d$, interparticle gap sizes $a$, and periodic boundary conditions along $x$ and $y$. To obtain thin slabs of an fcc crystal we stacked nanoparticle layers along $z$ in an $a b c$ stacking.

We used a mesh-override region with $0.5 \mathrm{~nm}$ mesh size for $a>1.5 \mathrm{~nm}$ and $a / 3$ mesh size for smaller gaps. The gold nanoparticles were modeled with the experimental dielectric function of gold from Ref. 44. For simulations within the Drude model we used the dielectric function $\varepsilon(\omega)=1-\omega_{p}^{2} /\left(\omega^{2}+i \omega \gamma_{p}\right)$ with $\hbar \omega_{p}=9.07 \mathrm{eV}$ and $\hbar \gamma_{p}=40 \mathrm{meV}$. We modeled the surrounding medium with a dielectric constant of $\varepsilon_{m}=1.96$ mimicking the experiment where the gold nanoparticles are surrounded with polystyrene molecules ${ }^{33}$.

To calculate the optical response of the crystal slab, we illuminated the structure with a broadband plane electromagnetic wave source along $z$ with linear polarization along $x$. We verified that the direction of polarization in the $x y$ plane has no influence on the calculated farfield spectra. The transmitted $T$ and reflected $R$ light were recorded with two-dimensional frequency-domain power monitors and the absorbance was calculated as $A=100 \%-T-R$. 
We calculated the normalized coupling strength for gold nanoparticle crystals in Fig. 1c from simulated absorbance spectra of gold nanoparticle films that consisted of six nanoparticle layers, see Extended Data Fig. 8. To simulate crystals with different coupling strength $\eta$, we either varied the nanoparticle diameters $d=10-100 \mathrm{~nm}$ for fixed $a=2 \mathrm{~nm}$, or varied the gap size $a=1-20$ $\mathrm{nm}$ for fixed $d=50 \mathrm{~nm}$. The simulated absorption spectra were analysed as described for the experimental data to obtain the simulated polariton dispersion. For spectra in which the absorbance peaks strongly overlapped we analyzed the internal current distribution as explained in Ref. 33. The polariton energies and wavelengths yield the polariton dispersions that are shown in Extended Data Fig. 8 as data points. We find the normalized coupling strength $\eta=\Omega_{R} / \omega_{p l}^{t}$ from a fit with the Hopfield model in Eq. (1). The resulting $\eta$ are the data points shown in Fig. 1c.

\section{The Hopfield and the Lorentz model}

The Hopfield model introduced with Eq. (1) represents a quantum mechanical formalism to describe the interaction of light and a material excitation. An alternative approach models the excitations through a classical Lorentz oscillator (or a series of oscillators). The Lorentz model yields a dielectric function that is specified by the excitation frequency $\omega_{p l}$, its line width $\gamma$ and reduced oscillator strength $f$, and the high-frequency dielectric constant $\varepsilon_{\infty}$

$$
\varepsilon_{L}(\omega)=\varepsilon_{\infty}+f \frac{\omega_{p l}^{2}}{\omega_{p l}^{2}-\omega^{2}-i \gamma \omega} .
$$

This is exactly the dielectric function of the Hopfield model $\varepsilon_{H}(\omega)$ as is obtained by substituting the dispersion relations $c^{2}|\mathbf{k}|^{2}=\varepsilon_{H} \omega_{p p}^{2}$ for the polariton and $c^{2}|\mathbf{k}|^{2}=n_{e f f}^{2} \omega_{p t}^{2}$ for the photon into Eq. (1) and setting $\omega \equiv \omega_{p p}{ }^{24,25}$. We include intrinsic losses by a complex frequency component $i \gamma \omega$.

Comparing the two dielectric functions $\varepsilon_{L}$ and $\varepsilon_{H}$ let us identify $f=4 n_{e f f}^{2} \Omega_{R}^{2} / \omega_{p l}^{2}=4 n_{e f f}^{2} \eta^{2}$ and $\varepsilon_{\infty}=n_{e f f}^{2}$. The reduced coupling strength is related to the oscillator strength of the transition. This useful connection simplifies a screening for materials and metamaterials in the ultrastrong and deep strong coupling regime. For example, excitons in semiconductors have typically $f \approx 10^{-4}-10^{-3}$ (Ref. 45 ); $\mathrm{ZnO}$ as a semiconductor with very high oscillator strength reaches $f=0.01^{46}$. Plasmonic metamaterials modeled within the effective medium theory have much higher oscillator strength, e.g., $f=2.7$ in Ref. 47. However, all materials fell at least an order of magnitude short of the maximum $f=16.6$ observed by us.

The dielectric function allows to calculate transmission and reflection spectra for thin slabs of plasmonic crystals that fully reproduce our optical spectra. The plasmonic crystal can be treated 
as an open cavity where radiative damping occurs through cavity losses ${ }^{22}$, in addition to the intrinsic loss $\gamma$. To calculate the optical spectra we treat the plasmonic crystal as a metamaterial of effective thickness $h$ with dielectric function $\varepsilon_{L}(\omega)$ [or $\left.\varepsilon_{H}(\omega)\right]$ and permeability $\mu_{\mathrm{pp}}=1$. The optical transmittance and reflectance are then given at normal incidence by ${ }^{48}$

$$
T=\left|\frac{4 n_{p p} e^{i h n_{p p} \omega / c}}{\left(1+n_{p p}\right)^{2}-\left(1-n_{p p}\right)^{2} e^{2 i h n_{p p} \omega / c}}\right|^{2},
$$

and

$$
R=\left|\frac{\left(1-n_{p p}^{2}\right)\left(1-e^{2 i h n_{p p} \omega / c}\right)}{\left(1+n_{p p}\right)^{2}-\left(1-n_{p p}\right)^{2} e^{2 i h n_{p p} \omega / c}}\right|^{2},
$$

where $n_{p p}=\sqrt{\varepsilon_{L}}=\sqrt{\varepsilon_{H}}$. The model was used to calculate the optical absorption spectra in Extended Data Fig. 7 as $A=100 \%-T-R$.

While the Hopfield and the Lorentz model are equally suited to describe our experimental observations, the Hopfield Hamiltonian gives insight into the physical processes of light-matter coupling that the Lorentz model lacks ${ }^{25}$. For example, the Hopfield Hamiltonian allows to calculate the light and matter fraction of polaritons, the ground state energy of the system, and the population with virtual excitations ${ }^{1}$. It also sets the framework for studying quantum effects in nanoparticle crystals, e.g., squeezed light, polariton condensation, and the generation of correlated photons from the quantum vacuum ${ }^{1,4,7,39,40}$.

\section{Microscopic model for the collective plasmons}

The plasmon polaritons of the nanoparticle crystals in Fig. 1 were calculated within the microscopic model of Lamowski et al. ${ }^{24}$. We stress that all quantitative statements in the paper as well as the data analysis were done with the Hopfield Hamiltonian using $\omega_{p l}^{t}$ and $\Omega_{R}$ as free parameters. The microscopic model illustrates how polaritons emerge in nanoparticle crystals starting from the dipole excitation of each sphere. In the following, we summarize the main features and findings of the microscopic description. The reader is referred to Ref. 24 for a full account.

The model considers an infinite three-dimensional crystal of plasmonic nanoparticles. The particles are described as spheres with diameter $d$, interparticle gaps $a$, and the dielectric function $\varepsilon(\omega)=\varepsilon_{\infty}-\omega_{p}^{2} / \omega^{2}$ of a Drude metal, where $\hbar \omega_{p}=9.07 \mathrm{eV}$ is the plasma frequency and $\varepsilon_{\infty}$ the dielectric screening by the electrons of the metal. We discuss the applicability of all approximations made in this microscopic model below. The nanoparticle dipole plasmon with frequency

$$
\omega_{N P}=\frac{\omega_{p}}{\sqrt{\varepsilon_{\infty}+2 \varepsilon_{m}}}
$$


is described as a point dipole in the center of a sphere with dielectric constant $\varepsilon_{\infty}$. This sphere is surrounded by a medium with dielectric constant $\varepsilon_{m}$. The Coulomb interaction between the plasmonic dipoles in the crystal creates collective plasmonic states described by a plasmonic band structure ${ }^{22,24}$, see Extended Data Fig. 1,

$$
\omega_{p l}(\mathbf{k})=\omega_{N P} \sqrt{1+g f(k)},
$$

with the coupling factor

$$
g=\frac{3}{4} \frac{3 \varepsilon_{m}}{\varepsilon_{\infty}+2 \varepsilon_{m}}\left(\frac{d}{d+a}\right)^{3} F_{0},
$$

where $F_{0}$ is the metal fill factor for vanishing gap size, see Supplementary Table S1. $f(\mathbf{k})$ is a dimensionless factor that depends on the crystal structure and plasmon polarization. It is calculated from the lattice sums of the plasmonic dipoles ${ }^{24}$. The plasmonic band structure consists of transverse $\omega_{p l}^{t}(\mathbf{k})$ and longitudinal branches $\omega_{p l}^{l}(\mathbf{k})$. The transverse plasmons $\omega_{p l}^{t}(\mathbf{k})$ couple strongly to light $\omega_{\mathrm{pt}}(\mathbf{k})$, resulting in hybridized plasmon polaritons $\omega_{p p}(\mathbf{k})$, as described in the main text.

The coupling factor $g$ may be related to the coupling of the transverse plasmon to the retarded photon field given by the Rabi frequency $\Omega_{R}=\omega_{N P} \sqrt{g}$. At first sight this appears surprising, but it results from the simple fact that both types of coupling (plasmon-plasmon and plasmon-photon) proceed through the dipole interaction.

From the model above we derive an analytic expression for the normalized coupling constant

$$
\eta=\frac{\Omega_{R}}{\omega_{p l}^{t}}=\left[\frac{\varepsilon_{\infty}+2 \varepsilon_{m}}{3 \varepsilon_{m}} \frac{4}{3 F_{0}}\left(\frac{d+a}{d}\right)^{3}+f_{t}(\mathbf{k})\right]^{-1 / 2}
$$

which was plot in Fig. 1c.

The point-dipole approximation is valid for any $\varepsilon_{\infty}$ and $\varepsilon_{m}$ if $a \geq d / 2$ or any $d$ and $a$ if $\varepsilon_{\infty}=\varepsilon_{m}$ $=1(d \geq 10 \mathrm{~nm}$ and $a \geq 1 \mathrm{~nm}$ as we neglect quantum size effects and electron tunneling between particles). In our modeling we used $a \ll d$ and $\varepsilon_{m}>1$ (1.96). To verify the quality of the pointdipole approximation under these conditions, we compared the polariton dispersions predicted by the analytic model with FDTD simulations. Extended Data Figure 9a shows the dispersion for $d=30 \mathrm{~nm}, a=2 \mathrm{~nm}, \varepsilon_{\infty}=1$, and $\varepsilon_{m}=1.96$, as used in Fig. 1. The analytic model agrees well with the numerical simulations, confirming that the point-dipole approximation may be employed for this range of parameters. Note that no free parameters were used in preparing Extended Data Fig. 9; we simply plot two independent calculations on top of each other. Close to the $\Gamma$ point, the energy of the upper polariton branch is underestimated by the analytic theory. The discrepancy disappears when changing $\varepsilon_{m}$ to one, Extended Data Fig. 9b. For completeness, Fig. 9c presents 
the perfect agreement of the analytic dispersion with FDTD data for $a>d / 2(d=30 \mathrm{~nm}, a=20$ $\mathrm{nm}, \varepsilon_{\infty}=1$, and $\left.\varepsilon_{m}=1.96\right)$.

A second aspect to consider are material losses. Although the analytic theory neglects losses, it excellently reproduces the polariton frequencies from FDTD simulations, where intrinsic and radiative losses are included. Losses do not affect the Rabi splitting and normalized coupling strength, but manifest in the spectral width of the peaks in the transmission, reflection and absorption spectra ${ }^{35}$. Losses can be included in the polariton dispersion with a complex frequency component, as shown in the Methods Section The Hopfield and the Lorentz model.

\section{Effect of crystal thickness on coupling strength}

The experimental polariton dispersions in Fig. 3d were obtained from the optical far field spectra of plasmonic crystals with varying thickness. When fitting the experimental data with the dispersion from the Hopfield Hamiltonian, we assumed that the coupling strength is independent of the crystal thickness. In this section we show with simulations that the normalized coupling strength decreases by less than $10 \%$ when decreasing the thickness from twenty to two nanoparticle layers. Larger differences (16\%) were observed in the experimental data, which we attribute to a decrease in the interparticle gap sizes with increasing crystal thickness.

Extended Data Figure 10a shows the simulated polariton dispersion for a gold nanoparticle crystal with $d=50 \mathrm{~nm}$ and $a=2 \mathrm{~nm}$. The data points were obtained from fits of the absorbance peaks in the simulated spectra and are color coded by the number of nanoparticle layers. The black line is a fit of the data points for 20 layers with the Hopfield dispersion [Eq. (1)], from which we obtain $\eta=1.59, \hbar \Omega_{R}=2.84 \mathrm{eV}$ and $\hbar \omega_{p l}^{t}=1.79 \mathrm{eV}$. While the data points for ten layers nicely track the fit for twenty layers, we observe a systematic shift of the data points for smaller layer numbers to higher energies. To analyze the coupling strength for smaller layer numbers, we reduce the fitting parameters from $\Omega_{R}$ and $\omega_{p l}^{t}$ to $\Omega_{R}$ by setting $\omega_{p l}^{t}=\sqrt{\omega_{N P}^{2}+\Omega_{R}^{2} f_{t}}$, using $\hbar \omega_{N P}=2.2 \mathrm{eV}$ from Mie theory and $f_{t}=-0.21$ from the fit for twenty layers. The normalized coupling strength drops with decreasing layer number by $8 \%$ to $\eta=1.45$ for a bilayer (see inset of Extended Data Fig. 10a).

We repeated this analysis for the experimental data of the gold nanoparticle crystals with $d=$ $41 \mathrm{~nm}$ (Extended Data Fig. 10b). The deviation of the data points from the Hopfield fit was most significant for this data set (compare blue dots and blue line in Fig. 3d). The black line in Extended Data Fig. 10b is a fit of the data points for five to seven layers. Similar to the simulations in Extended Data Fig. 10a, the data points for smaller layer numbers systematically shift to larger energies. The normalized coupling strength remains almost constant for four to 
seven layers $(\eta=1.33$ for $4 \mathrm{~L}$ and $\eta=1.37$ for $7 \mathrm{~L})$ and drops significantly to $\eta=1.14$ for a bilayer (see inset of Extended Data Fig. 10b). The decrease of the normalized coupling strength in the experiment $(16 \%)$ is larger than in the simulations $(8 \%)$. We believe this is due to a decrease of the interparticle gap sizes with increasing thickness of the gold nanoparticle crystals in our experiments.

The reduction in coupling strength for small layer numbers leads to an underestimation of $\eta$ in Table 1, especially for the gold nanoparticle crystals with $d=46 \mathrm{~nm}$ where only $2 \mathrm{~L}-4 \mathrm{~L}$ were analyzed. For the gold nanoparticle crystals with $d=41 \mathrm{~nm}$ the coupling strength is $\eta=1.37$ for seven layers, while a fit of the data points for all layers yields $\eta=1.31$. The deviation is less than five percent. We conclude that, except for the $d=46 \mathrm{~nm}$ gold nanoparticle crystals, the uncertainty of $\eta$ in Table I remains on the order of $10^{-2}$.

[41] Bastús, N. G., Comenge, J. \& Puntes, V. Kinetically controlled seeded growth synthesis of citrate-stabilized gold nanoparticles of up to $200 \mathrm{~nm}$ : Size focusing versus Ostwald ripening. Langmuir 27, 11098-11105 (2011).

[42] Zheng, Y., Zhong, X., Li, Z. \& Xia, Y. Successive, seed-mediated growth for the synthesis of single-crystal gold nanospheres with uniform diameters controlled in the range of $5150 \mathrm{~nm}$. $P$. \& P. Sys. Charact. 31, 266-273 (2014).

[43] Dong, A., Chen, J., Vora, P. M., Kikkawa, J. M. \& Murray, C. B. Binary nanocrystal superlattice membranes self-assembled at the liquid-air interface. Nature 466, 474 (2010).

[44] Olmon, R. L. et al. Optical dielectric function of gold. Phys. Rev. B 86, 235147 (2012).

[45] Yu, P. \& Cardona, M. Fundamentals of Semiconductor Physics (Springer, Heidelberg, 1996). [46] Mihailovic, M. et al. Optical and excitonic properties of ZnO films. Opt. Mat. 31, 532 (2009). [47] Liu, N. et al. Three-dimensional photonic metamaterials at optical frequencies. Nat. Mat. 7, 31 (2007).

[48] Nicolson, A. M. \& Ross, G. F. Measurement of the intrinsic properties of materials by timedomain techniques. IEEE Trans. Instrum. Meas. 19, 377-382 (1970).

[49] Rakíc, A. D. Algorithm for the determination of intrinsic optical constants of metal films: Application to aluminum. Appl. Opt. 34, 4755-4767 (1995).

Data Availability: All data is available in the manuscript and the Supplementary Information. 


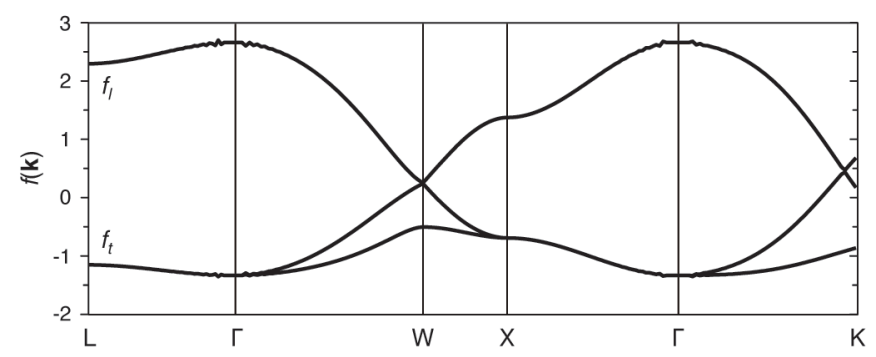

Extended Data Fig. 1. Plasmon dispersion without light-matter interaction calculated with a microscopic theory that is based on the point-dipole approximation and the Drude $\operatorname{model}^{24}$, see Methods. The following parameters were used: fcc crystal, nanoparticle diameter $d=30 \mathrm{~nm}$, interparticle gap size $a=2 \mathrm{~nm}, \hbar \omega_{p}$ $=9.07 \mathrm{eV}, \varepsilon_{\infty}=1$, and $\varepsilon_{m}=1.96$. The plasmonic band structure was calculated with $\omega_{p l}(\mathbf{k})=$ $\omega_{N P} \sqrt{1+\mathrm{g} f(\mathbf{k})}$, where we set $f_{t}=-4 / 3$ and $f_{l}=8 / 3$ close to the $\Gamma$ point because of a divergence of the lattice sums; see discussion in Ref. 24. The oscillations close to $\Gamma$ are an artefact of the calculations and were omitted when calculating the polariton dispersion in Fig. $1 \mathrm{~b}$. 

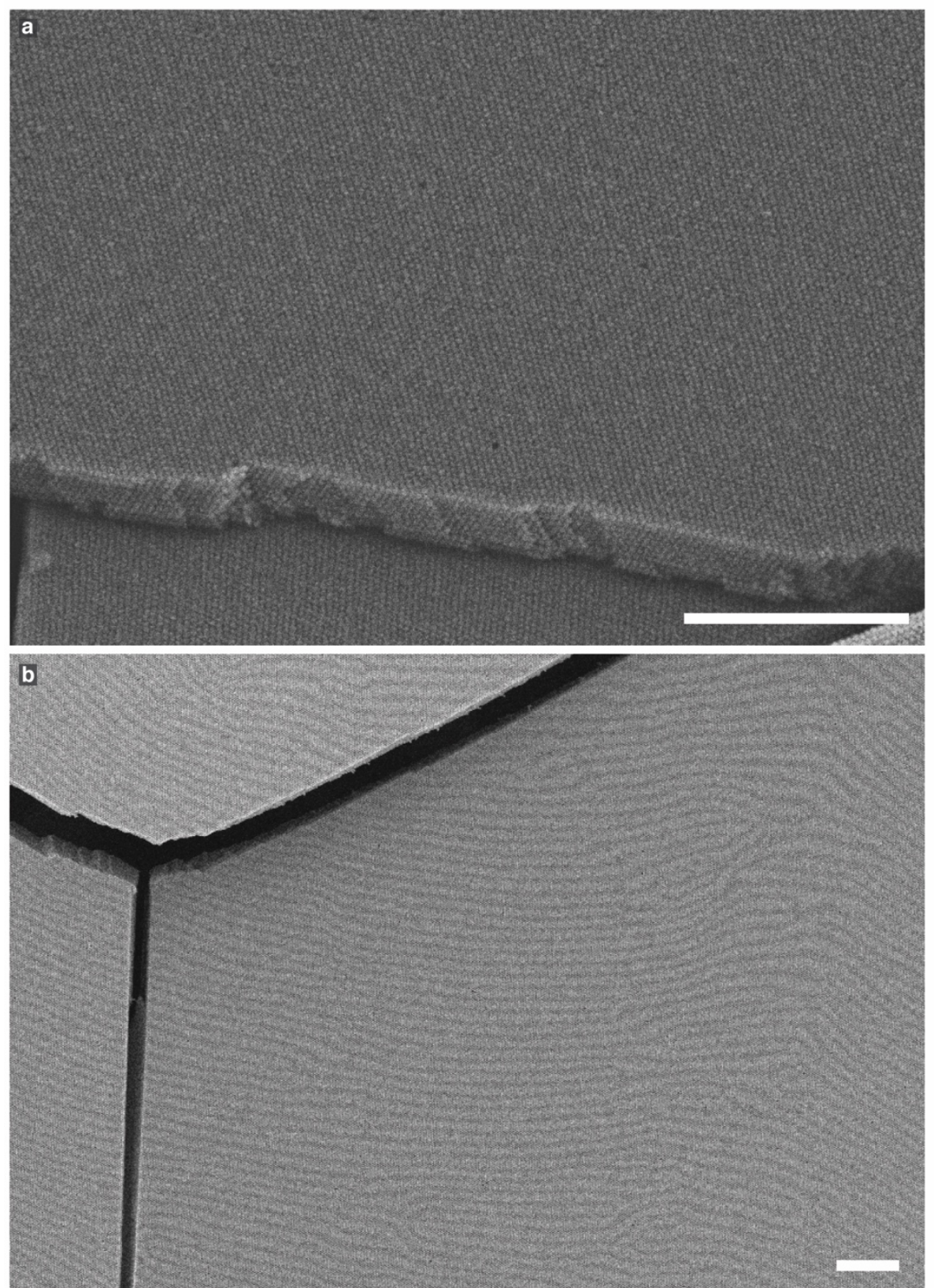

Extended Data Fig. 2. SEM images of gold nanoparticle crystals showing large crystallites. (a) Gold nanoparticle crystal that consists of $30 \mathrm{~nm}$ gold nanoparticles. (b) Gold nanoparticle crystal that consists of $59 \mathrm{~nm}$ gold nanoparticles. The scale bars are $1 \mu \mathrm{m}$. 


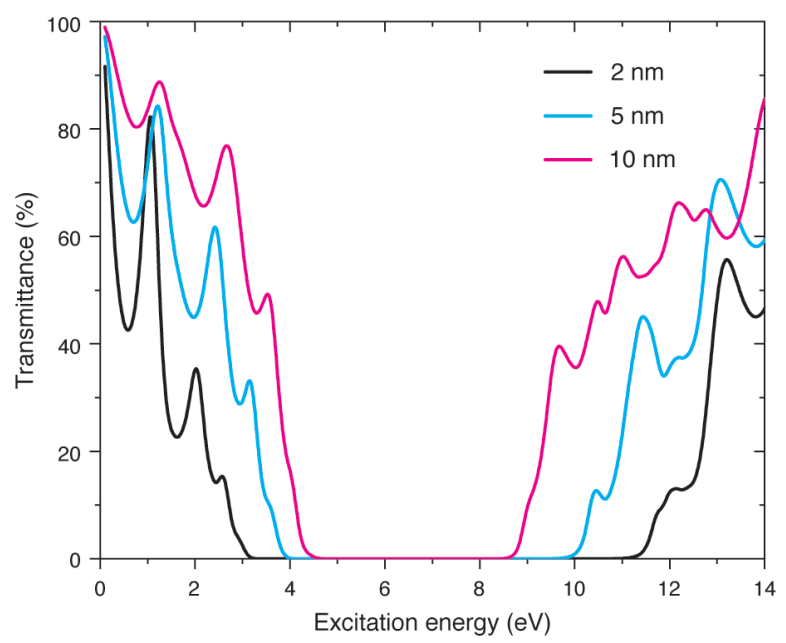

Extended Data Fig. 3. Simulated optical transmission of aluminium nanoparticle fec crystals that consist of six layers of $d=30 \mathrm{~nm}$ nanoparticles with $a=2 \mathrm{~nm}$ (black), $5 \mathrm{~nm}$ (cyan), and $10 \mathrm{~nm}$ (magenta) gaps. The dielectric constant of the embedding medium was set to $\varepsilon_{m}=1.96$ and the optical properties of aluminium were modeled with a fit of the experimental data from Rakíc ${ }^{49}$.
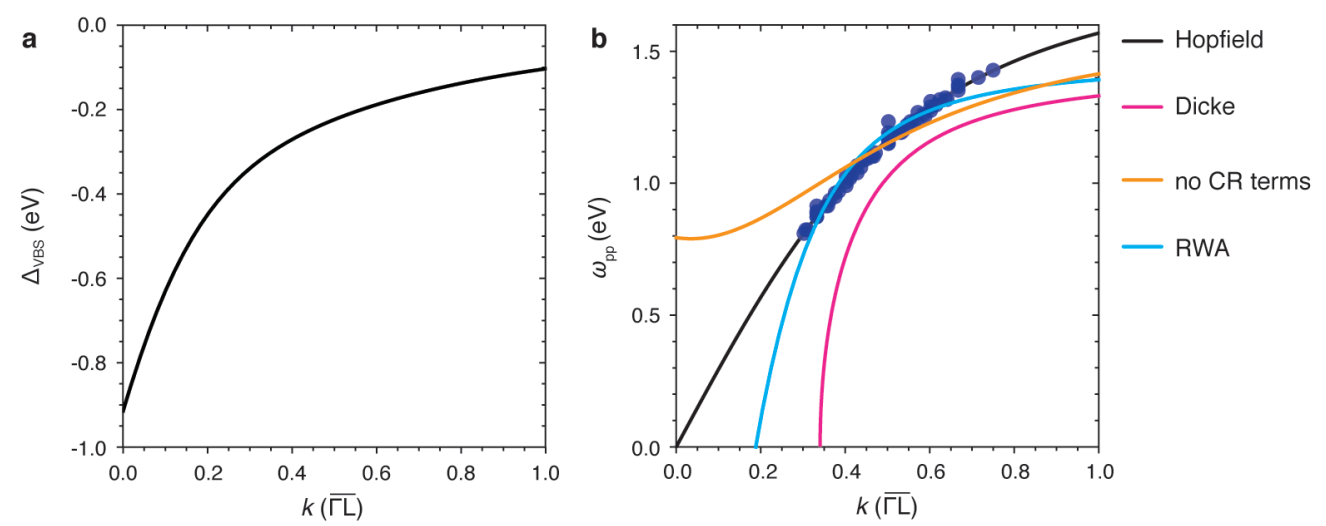

Extended Data Fig. 4. Approximations of the Hopfield Hamiltonian. (a) Vacuum Bloch-Siegert shift $\Delta_{V B S}$ calculated from the difference between the polariton dispersion of the full Hamiltonian (black line in Fig. 4a) and the dispersion of the Hamiltonian when neglecting all counter rotating terms (orange line in Fig. 4a). (b) Fitting experimental data $(d=59 \mathrm{~nm})$ with the polariton dispersions of the interaction Hamiltonians discussed in the Supplementary Information. The black line is a fit with the Hopfield model, Eq. (S8). The pink line shows a fit with the polariton dispersion when neglecting the $A^{2}$ term in the interaction Hamiltonian [Dicke model, Eq. (S9)]. The orange line is a fit with the dispersion when neglecting all counter-rotating terms [no CR terms, Eq. (S11)]. The blue line is a fit with the RWA approximation [no counter-rotating terms and no $A^{2}$ term, Eq. (S12)]. 


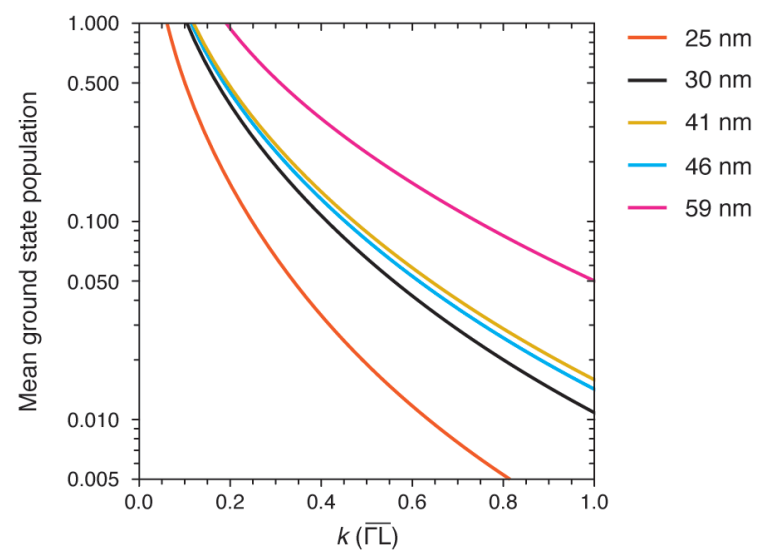

Extended Data Fig. 5. Mean population of the ground state with virtual photons $N_{p t}^{g}$ and virtual plasmons $N_{p l}^{g}$ as a function of wave vector $k$. The populations were calculated from the Hopfield model following Ref. 1 for the experimental nanoparticle crystals in Fig. 3. We used the fitting parameters from Table I.

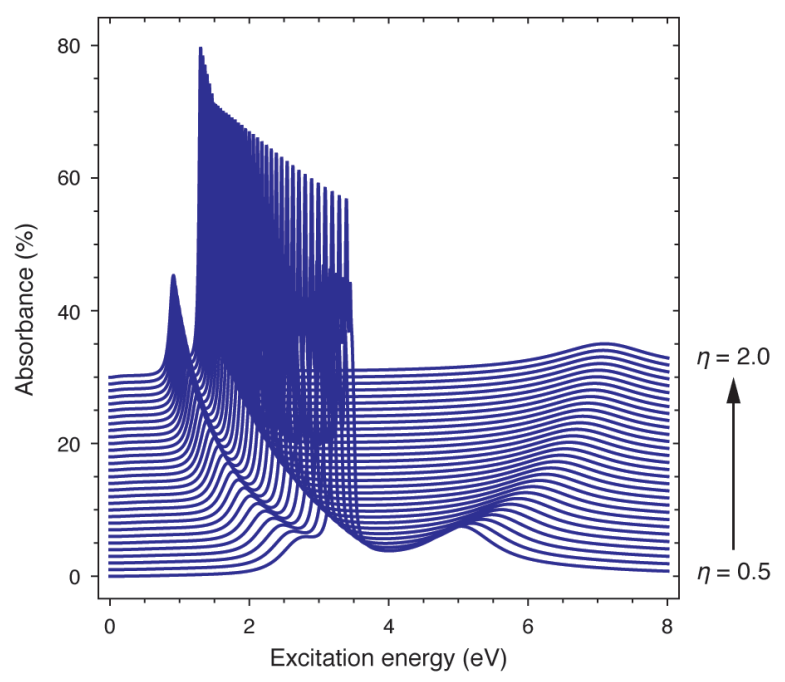

Extended Data Fig. 6. Absorption spectra calculated from the Hopfield Hamiltonian when going from ultrastrong to deep strong coupling. The absorption by the lower polariton branch increases in intensity and decreases in line width, vice versa for the upper polariton branch. The normalized coupling strength $\eta=0.5-2.0$ varies in increments $\Delta \eta=0.05\left(\hbar \omega_{N P}=4.06 \mathrm{eV}, \omega_{p l}^{t}=\sqrt{\omega_{N P}^{2}-4 \Omega_{R}^{2} / 3}\right.$, slab thickness of $100 \mathrm{~nm}$, and $\gamma=5 \cdot 10^{13} \mathrm{rad} / \mathrm{s}$ ). The model is discussed in the Methods Section. 

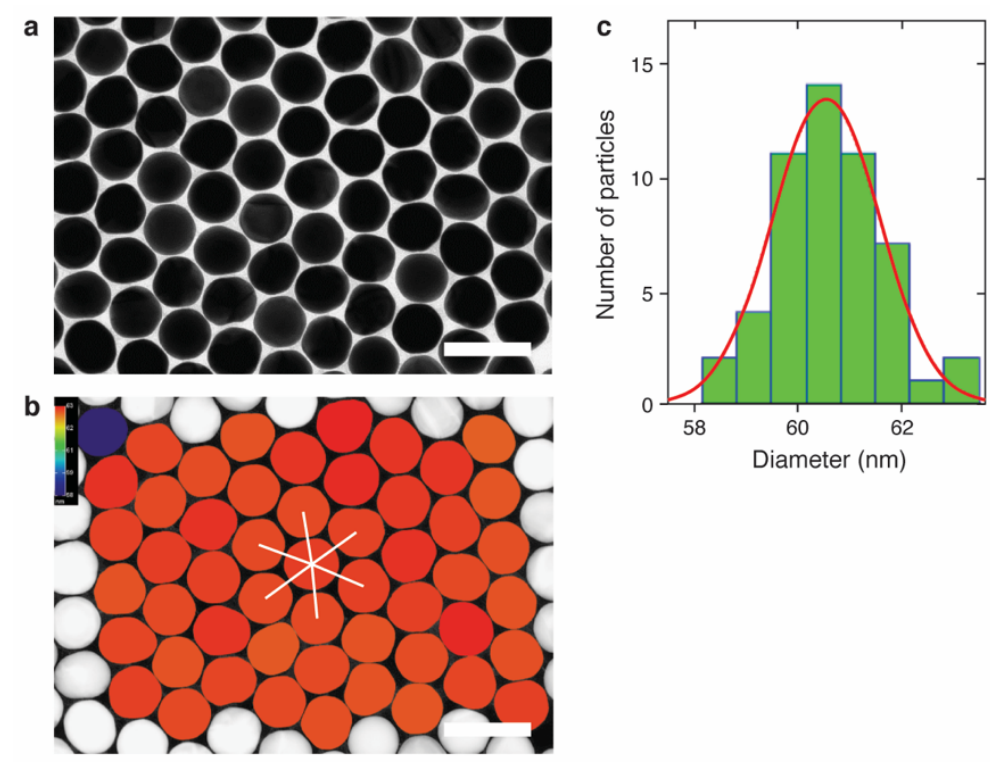

Extended Data Fig. 7. Evaluation of nanoparticle diameter $d$ and interparticle gap $a$ using a TEM image of a $59 \mathrm{~nm}$ nanoparticle monolayer. (a) TEM image of a monolayer of gold nanoparticles. (b) Evaluation of nanoparticle sizes and interparticle gap sizes of the same image as in (a) using the software Image J. The software determines the size of the nanoparticles (colored areas) giving the mean nanoparticle diameter. The interparticle gaps sizes were obtained by subtracting the particle diameters from the nearestneighbor distances (white lines). (c) Histogram of the nanoparticle diameters and fit with a Gaussian distribution. The mean values from the analysis of the TEM image in (a) are $d=60.6 \mathrm{~nm}$ and $a=1.5 \mathrm{~nm}$. The scale bars are $100 \mathrm{~nm}$.
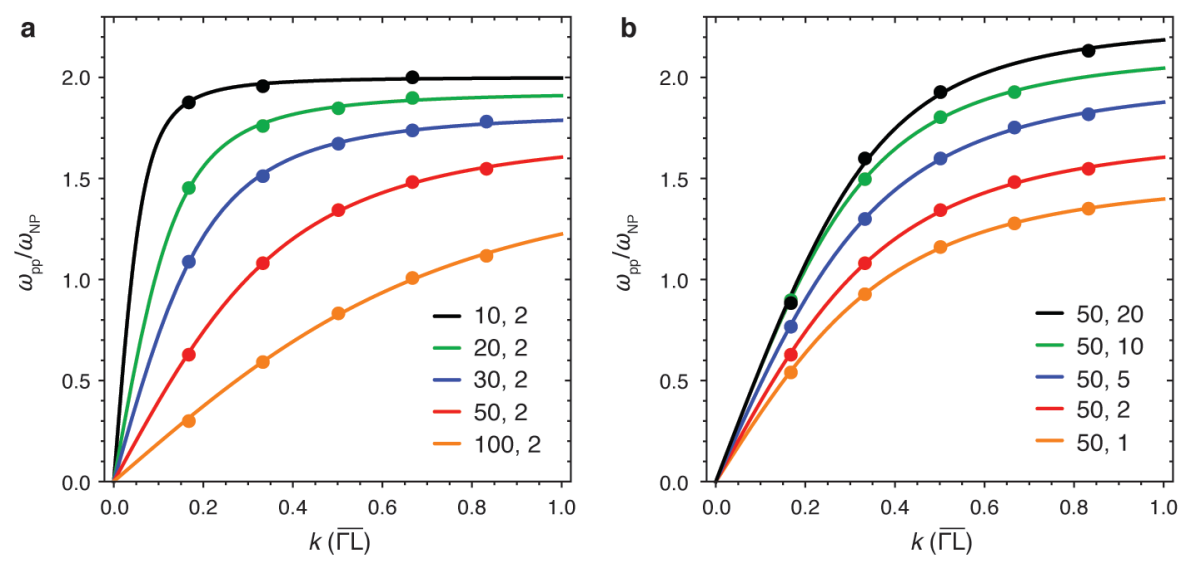

Extended Data Fig. 8. Simulated plasmon-polariton dispersions for gold nanoparticle fcc crystals with different diameters $d$ and interparticle gap sizes $a$ as specified by the labels $d, a$ (in nm). (a) Dispersions for different nanoparticle diameters and interparticle gap sizes of $2 \mathrm{~nm}$. (b) Dispersions for different gap sizes and nanoparticle diameters of $50 \mathrm{~nm}$. The data points were obtained from simulated absorbance spectra and the solid lines are a fit with Eq. (1) using $\Omega_{R}$ and $\omega_{p l}^{t}$ as fitting parameters. 

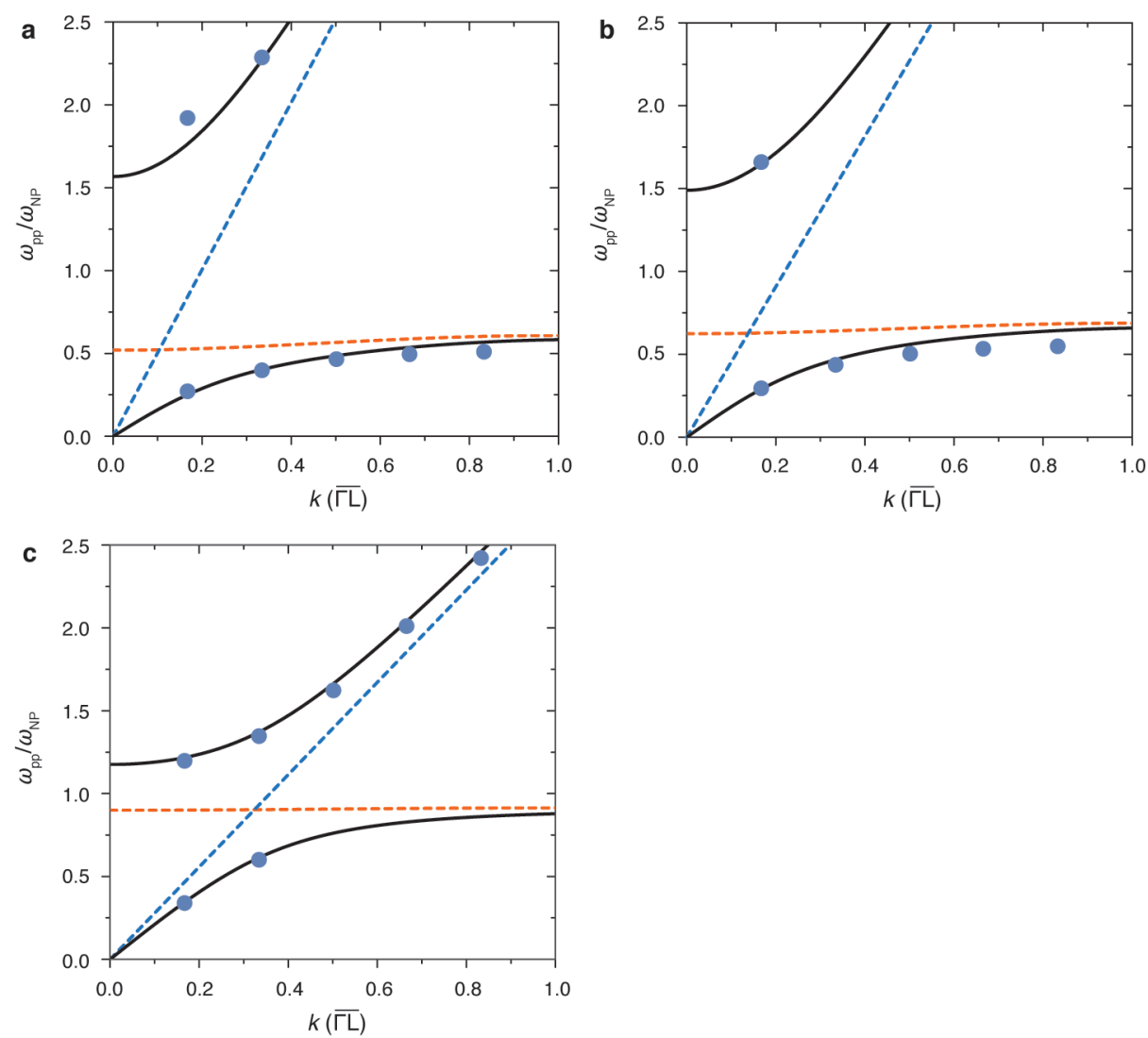

Extended Data Fig. 9. Comparison - not fit - of polariton dispersions from the microscopic model (solid black lines) and FDTD simulations (data points). (a) Same polariton dispersion as in Fig. 1b of the main paper, i.e., $d=30 \mathrm{~nm}, a=2 \mathrm{~nm}$ and $\varepsilon_{m}=1.96$. (b) Parameters as in (a) except for $\varepsilon_{m}=1$. (c) Polariton dispersion for $d=30 \mathrm{~nm}, a=20 \mathrm{~nm}$ and $\varepsilon_{m}=1.96$. We used the Drude model as the dielectric function of the metallic nanoparticles with $\varepsilon_{m}=1, \hbar \omega_{p}=9.07 \mathrm{eV}$ and $\hbar \gamma_{\mathrm{p}}=40 \mathrm{meV}$.
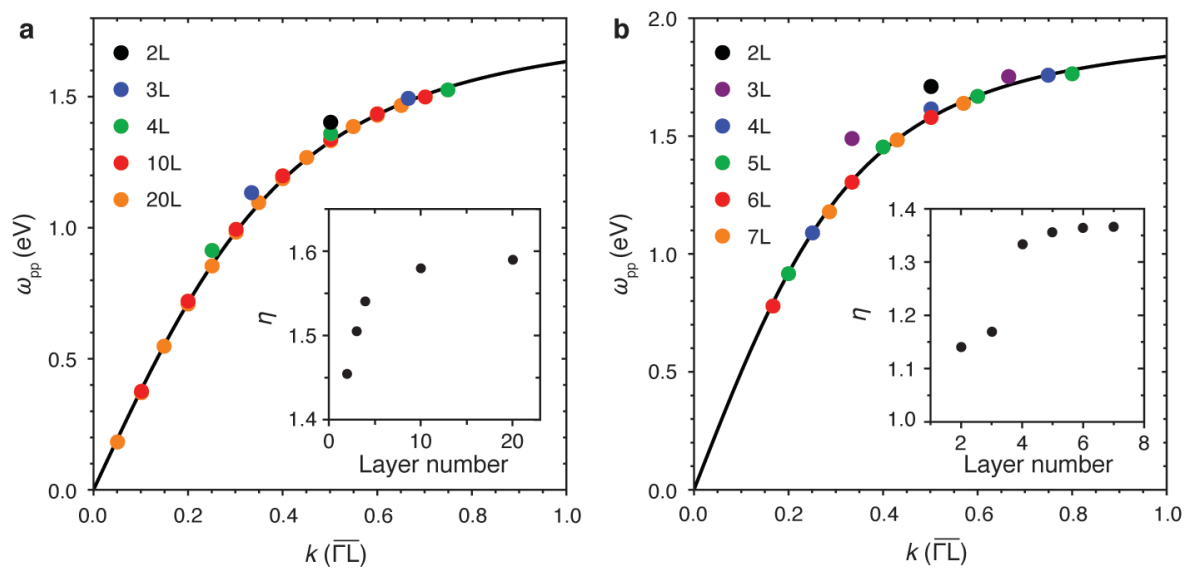

Extended Data Fig. 10. Effect of crystal thickness on the coupling strength. (a) Simulated polariton dispersion for an fcc gold nanoparticle crystal with $d=50 \mathrm{~nm}, a=2 \mathrm{~nm}$ and $\varepsilon_{m}=1.96$. The data points were obtained by fitting the absorption spectra of crystals with different layer number. The black line is a fit of the data points for 20 layers with the Hopfield dispersion, Eq. (1). The inset shows the normalized coupling strength as a function of layer number. (b) Same analysis as in (a) but for the experimental data 
of the gold nanoparticle crystal with $d=41 \mathrm{~nm}$. The black line is a fit of the data points for five to seven layers. 